\title{
On the dynamics of equatorial currents, with application to the Indian Ocean
}

\author{
Mark A. Cane*† \\ (Received 30 July 1979; accepted 12 December 1979: final revision received 8 February 1980)
}

\begin{abstract}
The dynamics of equatorial currents are considered in the light of the wind and current measurements at Gan $\left(0.5^{\circ} \mathrm{S}, 73^{\circ} \mathrm{E}\right)$ reported by KNox (Deep-Sea Research, 23, 211 221, 1976). For westerly and meridional winds the response of equatorial currents is predominantly local and rapid. It follows that the current reversals observed at Gan are most likely due to changes in the local winds and not to a reflection from the eastern boundary, as was previously suggested. The winds at Gan are predominantly westerly, resulting in downwelling at the equator. The eastward momentum put in by the wind at the surface is advected downward giving subsurface eastward flow. With persistent easterlies (as in the Atlantic and Pacific) there is an undercurrent driven by the eastward pressure gradient force, both because of the direct, down gradient flow and because of the vorticity transported by the associated meridional circulation. It is suggested that the undercurrent that has been observed in the Indian Ocean in the early spring is similarly driven by an eastward pressure force. This pressure gradient is a non-local transient feature generated by the zonal readjustment of mass induced by the relaxation of the winds at the fall monsoon transition. This idea is consistent with the presence of the undercurrent in 1973 and its absence in 1974.
\end{abstract}

\section{INTRODUCTION}

Figure 1, adapted from KNOX (1976), shows winds $\tau$, surface currents $U_{s}$ and thermocline depth currents $U_{t h}$ recorded near Gan $\left(0.5^{\circ} \mathrm{S}, 73^{\circ} \mathrm{E}\right)$. This is the longest time series of equatorial current observations available for the Indian Ocean, and it exhibits a number of interesting features. As noted by KNOX (1976), the surface current is highly correlated with the local wind with little or no phase lag (current measurements are no more frequent than once a week). Perhaps this is not surprising, but it is noteworthy that the subsurface currents also correlate with the local wind with little or no phase lag: an eastward $U_{t h}$ appears (disappears) rapidly with the switch on (off) of westerly winds. It suggests that the observed currents are primarily responding to local winds. In particular, the reversal of the currents in June--July is a local response to the changing winds rather than the result of an eastern boundary reflection, as proposed by KNOX (1976). (Note also that in the highly nonlinear calculation of CANE (1979a) the eastern boundary reflection does not reverse the flow on the equator as it does in the linear case or in the nearly linear calculation of O'Brien and Hurlburt (1974) referred to by Knox.)

March 1973 is the only time the record shows an undercurrent similar to that apparently always present in the Atlantic and Pacific. That is, an eastward subsurface flow of greater intensity than the (usually westward) surface flow. In this the Gan data are consistent with

* Present address: Department of Meteorology, Massachusetts Institute of Technology, Cambridge, MA 02139, U.S.A.

† Laboratory for Atmospheric Sciences, NASA Goddard Space Flight Center, Greenbelt, MD 20771, U.S.A. 


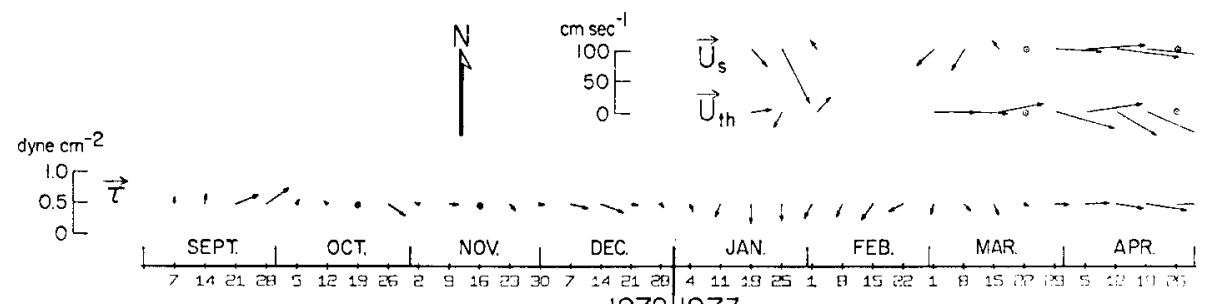
1972,1973
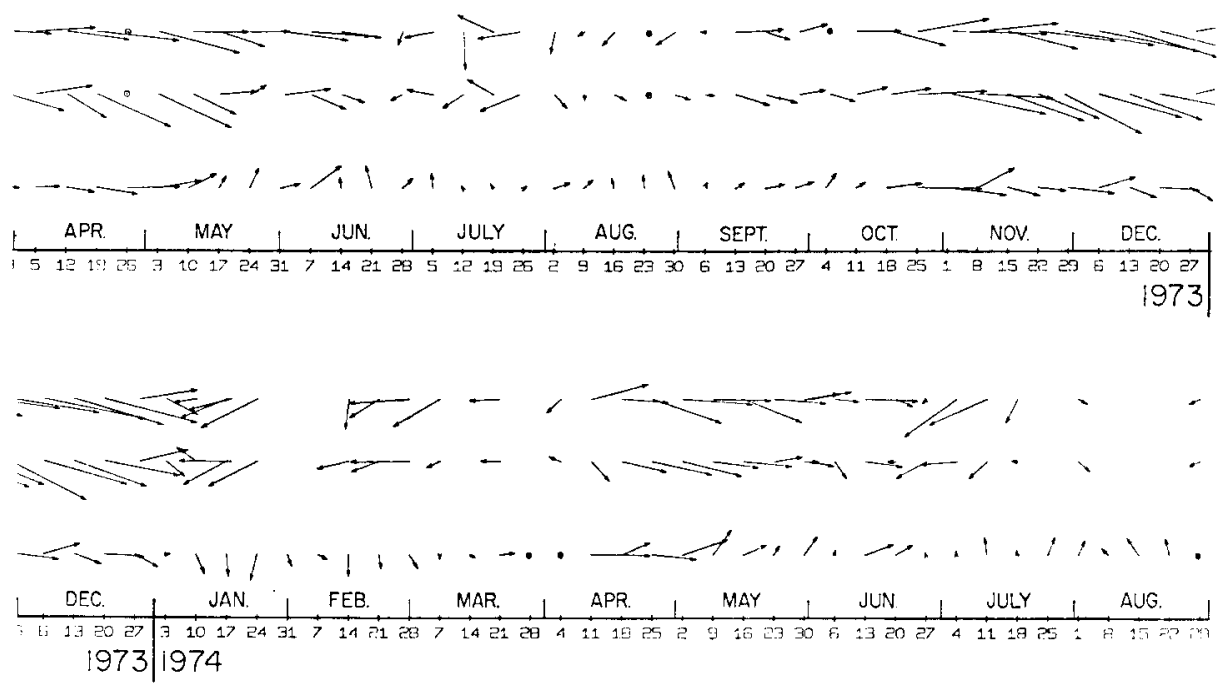

Fig. 1. Adapted from KNox (1976). Surface currents $u_{s}$ (averaged from 0 to $20 \mathrm{~m}$ ), thermocline currents $u_{t h}$ (averaged from 60 to $80 \mathrm{~m}$ ), and wind stress $\tau$ measured near Gan $\left(0.5^{\circ} \mathrm{S}, 73 \mathrm{E}\right)$. Open circles are unuseable data; closed circles are zero values.

the other observations of equatorial currents in the Indian Ocean (TAFT and KNAUSS, 1967 ; Swallow, 1967 ; also see Philander, 1973); the only time such an undercurrent has been observed is in the early spring (late February to early April). It is curious that such an undercurrent was absent in 1974. KNox (1976) suggested that the difference between 1973 and 1974 is largely attributable to the difference in the northeast winds in the early months of the year. These differences seem to be too slight and short-lived to be significant. Later, we suggest that it is the wind differences in the late months of the preceding year (also noted by Knox) that account for the undercurrent differences.

The remainder of this paper elucidates dynamical mechanisms that may account for the behavior observed near Gan. While the data set is unusually valuable because it is a long uninterrupted time series, it is still a single point in a large and largely unexplored ocean. For this reason we can only describe dynamics consistent with the observations; our knowledge of the Indian Ocean winds and currents is too limited for us to assert that these dynamics explain the observations.

Throughout this work we contrast the oceanic response at the equator to easterly and westerly winds. The difference in prevailing wind direction is the principal difference between the winds over the Atlantic and Pacific on the one hand and the Indian Ocean on 
the other. (A secondary difference is the degree of persistence of the winds.) As we will show, the degree to which the oceanic response may be considered local or nonlocal is a consequence of the difference in wind direction. A necessary corollary of this statement is that the response is essentially nonlinear. A linear ocean would change the sign of its response with a change in wind direction, but the response to easterlies and westerlies would be qualitatively the same.

The plan of the remainder of this paper is as follows: The next section discusses the basic dynamics governing the equatorial undercurrent and clarifies the reason for expecting differences in the response to easterlies and westerlies. Section 3 briefly describes the numerical model to be used in Section 4 to calculate the time evolution of the currents in response to switched on easterlies and westerlies. Section 5 discusses the changes in the currents that occur when the winds are relaxed and Section 6 considers the applicability of the results to the Indian Ocean.

\section{BASIC DYNAMICS}

The essential dynamics of the undercurrent were first explained by FofONOFF and MONTGOMERY (1955) and have since been elaborated by many investigators (CHARNEY, 1960; Charney and Spiegel, 1971; Robinson, 1966; Gill, 1975; Cane, 1979a, b). For a flow in which longitudinal and vertical variations are negligible compared to meridional ones the statement of conservation of vorticity reduces to

$$
\frac{\mathrm{d}}{\mathrm{d} t}\left(\beta y-u_{y}\right)=0
$$

where, as usual, $\beta=\mathrm{d} f / \mathrm{d} y$ with $f$ the Coriolis parameter, $u$ is zonal and $v$ meridional velocity and, with the stated assumptions, $\mathrm{d} / \mathrm{d} t=\partial / \partial t+v \partial / \partial y$. For steady flow (1) reduces to

$$
v \frac{\partial}{\partial y}\left(\beta y-u_{y}\right)=0 .
$$

For a parcel of fluid starting at latitude $y_{0}$ that reaches the equator we can integrate twice to conclude

$$
\beta y-u_{y}=\text { const. }=\beta y_{0}-u_{y}\left(y_{0}\right) \approx \beta y_{0}
$$

and

$$
u(y=0) \approx u\left(y_{0}\right)+\frac{1}{2} \beta y_{0}^{2}>0,
$$

where we have assumed that the initial zonal velocity and shear are negligible. [Equation (3) gives $u(y=0)=1 \mathrm{~m} \mathrm{~s}^{-1}$ for $y_{0}=2.7^{\circ}$.] As a parcel moves equatorward its loss of planetary vorticity is compensated by a gain of relative vorticity. Regardless of its hemisphere of origin the result is eastward flow at the equator.

The argument may obscure the difference between easterly and westerly winds. In Section 4 the early and the later stages of the response will be discussed separately. The distinction between the two stages is made on the basis of whether or not a zonal pressure gradient has been established. For now we focus on the later stage, appropriate to the steady state circulation considered above. The situation for this later stage is shown schematically in Fig. 2. 


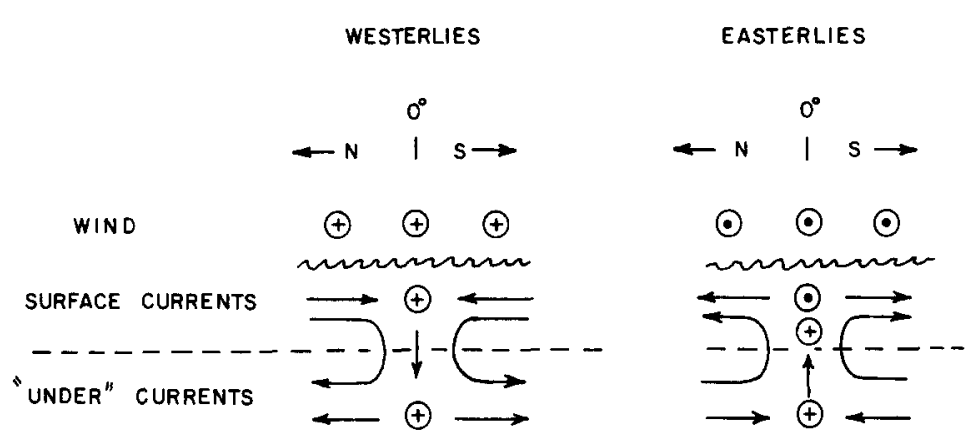

Fig. 2. Sketch of meridional section of the steady, long time response to easterlies and westerlies. Looking eastward.

With easterlies the wind drives a westward surface flow at the equator. The meridional Ekman flow is poleward and thus divergent at the equator resulting in strong upwelling. For an evolving flow a rising thermocline could supply the mass flux demanded by this upwelling, but a steady state requires equatorward flow at depth, as shown in Fig. 2 . The vorticity argument given above therefore implies that this equatorward flow will result in an eastward undercurrent at the equator. To drive this equatorward current an eastward pressure force at depth is required. In addition, right on the equator this force is a direct source of eastward momentum. Thus for easterlies the pressure gradient plays the primary role in maintaining the undercurrent. Such a pressure gradient is a non-local effect ; without meridional coasts the average zonal pressure gradient would be zero.

The point can be further clarified by considering the changes in angular momentum of a parcel moving equatorward. If we start with the inviscid zonal angular momentum equation

$$
\mathrm{d} u / \mathrm{d} t-f v=-\frac{1}{\rho_{0}} p_{x}
$$

and make the same assumptions as in the vorticity argument (i.e., $w=0$ and $\partial u / \partial x=\partial u / \partial t=0$ ) then the equation reduces to

or

$$
v\left(u_{y}-\beta y\right)=-\frac{1}{\rho_{0}} p_{x},
$$

$$
\beta y-u_{y}=\frac{1}{\rho_{0}} p_{x} / v .
$$

Using the initial condition that $u\left(y_{0}\right)=0$ this can be integrated to

$$
u(0)=-\frac{1}{2} \beta y_{0}^{2}+\int_{0}^{y_{0}} \frac{1}{\rho_{0}} p_{x} / v \mathrm{~d} y .
$$

If $p_{x}=0$ then angular momentum is conserved, in which case the parcel arrives at the equator moving westward relative to the earth. (This argument implies winds will be easterly at the equator; cf. HIDE, 1969). By comparing (2) with (4) we see that vorticity conservation implies that the right-hand side of (4) is constant and equal to $\beta y_{0}$ [again 
neglecting $u_{y}\left(y_{0}\right)$ ]. Using this in (5) again gives $u(0)=\frac{1}{2} \beta y_{0}^{2}$ : eastward flow at the equator. It is now clear how this result depends on the action of the pressure gradient force to increase the angular momentum of the parcel as it travels equatorward. It is interesting that the value of $u(0)$ is independent of $p_{x}$. It depends instead on the strength of the pressure gradient times the time it acts on the parcel and the greater $p_{x}$ the faster it moves (because $\left.v \propto p_{x}\right)$ and the less time $p_{x}$ has to turn the parcel eastward.

With westerlies the surface flow at the equator is driven eastward in the direction of the wind. The off-equator Ekman flow is equatorward in both hemispheres. implying enhanced eastward flow at the equator by the vorticity argument given above. However, frictional effects are certainly important in the surface layer and the vorticity effect is secondary to the direct wind driving. The important consequence of this Ekman drift is that it results in convergence at the equator and hence strong downwelling there. The eastward momentum in the surface layers is advected downward so that the subsurface flow is also eastward. This chain of reasoning demonstrates that the subsurface eastward flow is traceable to the surface layer flow, which is a (primarily frictional) response forced by the local wind. The description of the zero order dynamics makes no mention of the westward pressure gradient force that will be present as part of the steady state response. The force is a lower order influence that acts to retard the subsurface eastward flow at the equator. It does this both by its direct effect right at the equator and by its role in driving a subsurface meridional circulation that advects momentum away from the equator. (Poleward flow at depth clearly is required for mass conservation; geostrophy requires that the pressure gradient force be westward to help maintain such a flow.) Thus the response to westerlies has both local and non-local aspects. The local dynamics are responsible for the eastward flow at depth while the non-local westward pressure gradient force prevents this flow from continually accelerating.

\section{NUMERICAL MODEL}

The oceanic circulation will be calculated numerically, using a model much like the twolevel conceptual model introduced in the previous section. This model was derived by CANE (1979a) and used extensively by him (1979b); it will be described here only briefly. It has a surface layer of constant depth $\eta$ over which the wind stress is assumed to act as a body force. The layer thus has the same dynamical role as the mixed layer in the real ocean. There is an active layer below it extending to the main thermocline whose depth is determined dynamically. The upper layer velocity $u^{s}$ is the model's mixed-layer current and is comparable to $u_{s}$ of Fig. 1 while $u^{1}$ is the velocity averaged over the entire lower layer and so will generally be smaller than the thermocline value $u_{t h}$. The two active layers have the same constant density $\rho$ and communicate via a surface layer pumping $w_{e}$ as well as frictionally. The ocean below the thermocline is taken to be of density $\rho+\Delta \rho$ and is assumed to be at rest. Denoting surface layer quantities with superscripts and those in the lower active layer with superscript 1 the governing equations are

$$
\begin{aligned}
\mathbf{u}_{t}^{s}+\left(\mathbf{u}^{s} \cdot \nabla\right) \mathbf{u}^{s}=\frac{w_{e}}{2 \eta}\left(\mathbf{u}^{s}-\mathbf{u}^{1}\right)+f \mathbf{K} \times \mathbf{u}^{s} & =-g \frac{\Delta \rho}{\rho} \nabla h+\tau / \eta-\kappa / \eta\left(\mathbf{u}^{s}-\mathbf{u}^{1}\right)+v_{H} \nabla^{2} \mathbf{u}^{s}, \\
w_{e} & =\eta \nabla \cdot u^{s},
\end{aligned}
$$




$$
\begin{gathered}
\mathbf{u}_{t}^{1}+\left(\mathbf{u}^{1} \cdot \nabla\right) \mathbf{u}^{1}+\frac{w_{e}}{2 h}\left(\mathbf{u}^{s}-\mathbf{u}^{1}\right)+f \mathbf{K} \times \mathbf{u}^{1}=-g \frac{\Delta \rho}{\rho} \nabla h+\kappa / h\left(\mathbf{u}^{s}-\mathbf{u}^{1}\right)-\kappa / h \mathbf{u}^{1}+v_{H} \nabla^{2} \mathbf{u}^{1}, \\
h_{t}+w_{e}+\nabla \cdot\left(h \mathbf{u}^{1}\right)=0 .
\end{gathered}
$$

Notation is standard and all parameters have the same values as in CANE $(1979 \mathrm{a}, \mathrm{b}): \beta$ is constant, the magnitude of the wind stress is $4.7 \times 10^{-3} \mathrm{pa}, \eta=25 \mathrm{~m}$, the mean value of $h=175 \mathrm{~m}, \Delta \rho / \rho=0.0018, \quad v_{H}=60 \mathrm{~m}^{2} \mathrm{~s}^{-1}$, and the vertical friction parameter $\kappa=1.5 \times 10^{-5} \mathrm{~m} \mathrm{~s}^{-1}$. The basin extends from $15 \mathrm{~N}$ to $15 \mathrm{~S}$ and has a longitudinal extent of $28.6^{\circ}$. No slip conditions apply at the walls and the parameter values have been chosen so that vertical frictional processes dominate horizontal ones in the interior. The chosen value of $\kappa$ makes the magnitude of the model's vertical viscosity comparable to that in the world's equatorial oceans (or, at least, to our best guess at this value ; see CANE, 1979a, for a discussion of the observational evidence). Such a value means that for both the model and the oceans there is attenuation in the time it takes a Rossby wave to propagate across the basin.

\section{RESPONSE TO WINDS SWITCHED ON OVER A RESTING OCEAN}

In this section we consider the time evolution of currents in an equatorial ocean initially at rest. In all cases the winds are uniform in $x$ and $y$ and steady for $t>0$. We continue to contrast the response to easterlies with that to westerlies. Two distinct time periods will be considered: (a) the early stages of evolution, before coastal influences are felt so that $\partial / \partial x \equiv 0$, and (b) the later stages, when coastal effects are present so that $p_{x}$ is non-zero.

\section{(a) The early stages of evolution}

With a wind stress independent of longitude the ocean's response will be zonally uniform until such time as coastal influences introduce zonal variations. First consider a continuous ocean. With a zonal wind stress that is independent of latitude symmetry implies that $v=0$ at $y=0$. On the equator the zonal momentum equation simplifies to

$$
u_{t}=-w u_{z}+v_{v} u_{z z} \text {. }
$$

With a westerly wind stress $w$ is downward at the equator so both terms on the right-hand side of (7) are non-negative. Both diffusion and vertical advection act to transport eastward momentum downward. With easterlies there is upwelling but $u_{z}$ and $u_{z z}$ are both negative so that the first term is $\geqslant 0$ while the second is $\leqslant 0$. Westward momentum is diffused downward while vertical advection acts to remove westward momentum at depth. However, vertical advection has no initial effect because $u_{z}=0$ at a given depth until diffusion acts to make it otherwise. We therefore expect westward flow initially at all depths due to the dominance of the diffusion term. This expectation is borne out by PHILANDER's (1979) numerical calculation (see his Fig. 5).

By integrating (7) from the surface to a depth where the stress vanishes we obtain

$$
\frac{\partial}{\partial t} \int u \mathrm{~d} z=\tau^{(x)}+\int\left(-w u_{z}\right) \mathrm{d} z .
$$

In the linear case the last term is absent and the vertically integrated transport increases linearly in time in the direction of the wind. The inclusion of vertical momentum advection 
augments this transport for westerlies and decreases it for easterlies.

Turning now to the two-layer model it is again helpful in understanding the nonlinear effects to compare with linear results (Fig. 3). A thorough analysis of the linear behavior of the model is given in CANE (1979a). Neglecting horizontal diffusion the equation governing the surface layer velocity is, to lowest order in $\eta / h$,

$$
\mathbf{u}_{t}^{s}+f \mathbf{K} x \mathbf{u}=\left(\tau-\kappa \mathbf{u}^{s}\right) / \eta
$$

This equation is readily solved to yield, for a purely zonal wind stress, $\tau^{(x)}$

$$
u^{s}+i v^{s}=\frac{\tau^{(x)}}{\eta} \frac{(r-i f)}{r^{2}+f^{2}}\{1-\exp [-(r+i f) t]\},
$$

where $r=\kappa / \eta$ [ $=(11.6 \text { days })^{-1}$ for our parameter values $]$. Extra-equatorially this is just Ekman drift plus damped inertial oscillations; in the vicinity of the equator $\left(y \leqslant \beta^{-1} r\right)$ it is a zonal jet in the direction of the wind (see Figs $3 \mathrm{a}, \mathrm{b}$ ). Right on the equator $v^{s}=0$ and

$$
u^{s}=\left(\tau^{(x)} / \eta\right) r^{-1}\left[1-\mathrm{e}^{-r t}\right]
$$

so that initially $\left(t \ll r^{-1}\right) u^{s} \approx t \tau^{(x)} / \eta$. The boundary layer pumping (Fig. 3c) at the equator is given by

$$
w_{e}(y=0)=\eta v_{y}^{s}(y=0)=-\beta \tau^{(x)} r^{-2}\left[1-\mathrm{e}^{-r t}-r t \mathrm{e}^{-r r}\right] .
$$

Initially $w_{e}(y=0)=-\beta \tau^{(x)} t^{2}$ so that it increases rapidly and is independent of the value of the friction parameters (ultimately, i.e., for $t>r^{-1}, w_{e} \approx-\beta \tau^{(x)} r^{-2}$ and this is not the case). As we shall see, the vertical velocity at the equator plays an important role in determining the nonlinear response.

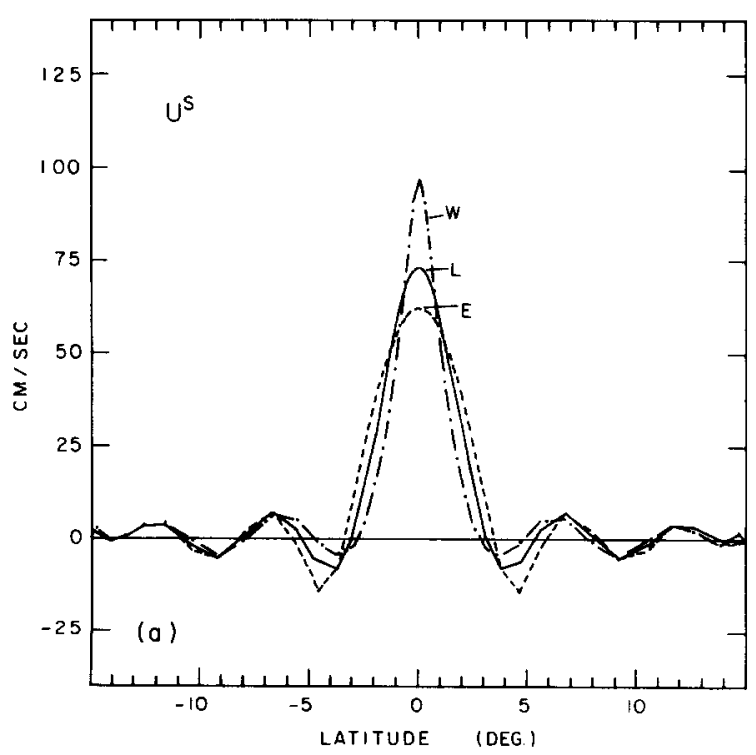

Fig. 3. Meridional sections of the $x$-independent model response to switched on winds: nonlinear, westerlies $(W)$; linear, westerlies $(L)$; the negative of the non-linear response to easterlies $(E)$. Shown at three equatorial time units (5.33 days). (a) Surface layer zonal velocity $u^{s}$. 


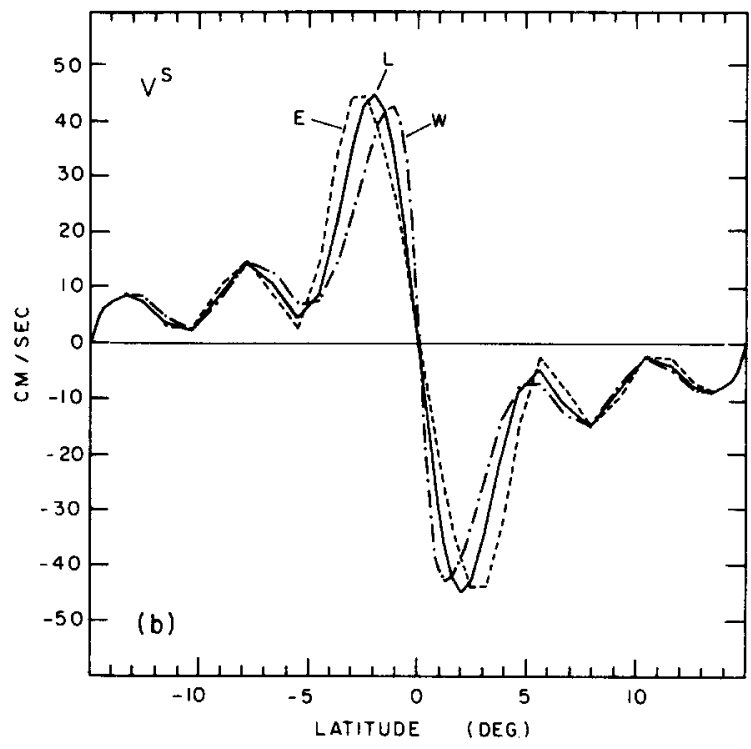

Fig. 3b. Surface layer meridional velocity, $v^{s}$.

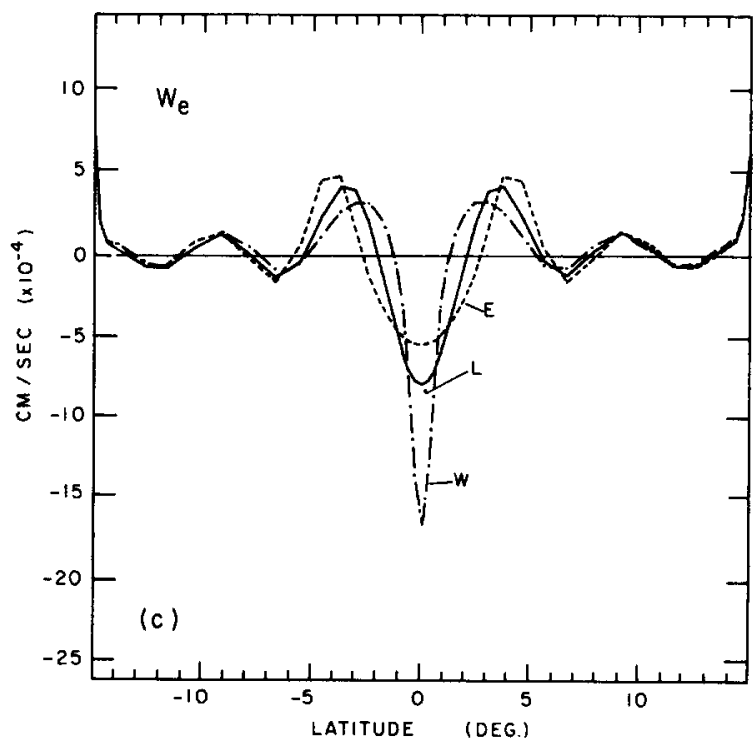

Fig. 3c. Vertical velocity, $w_{e}$. 


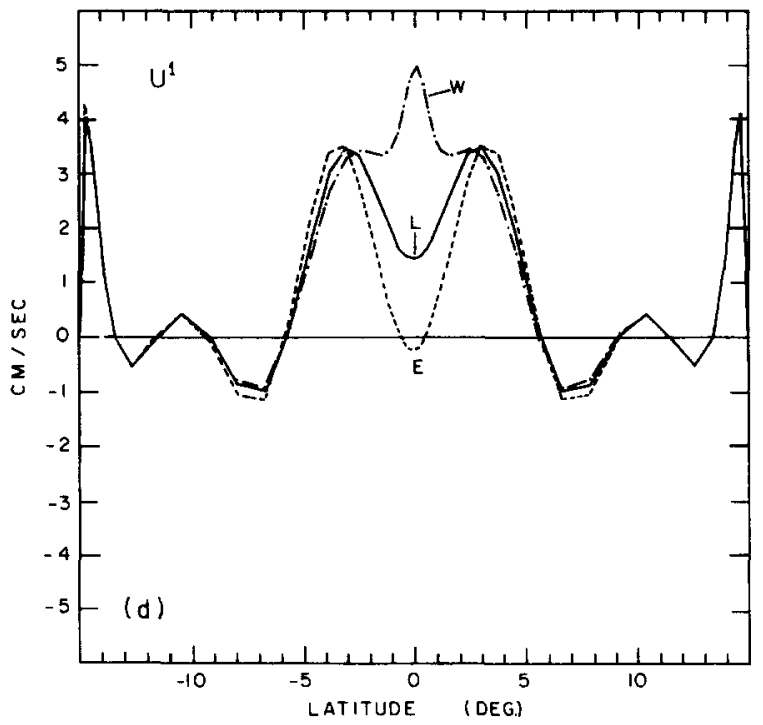

Fig. 3d. Lower layer zonal velocity, $u^{1}$.

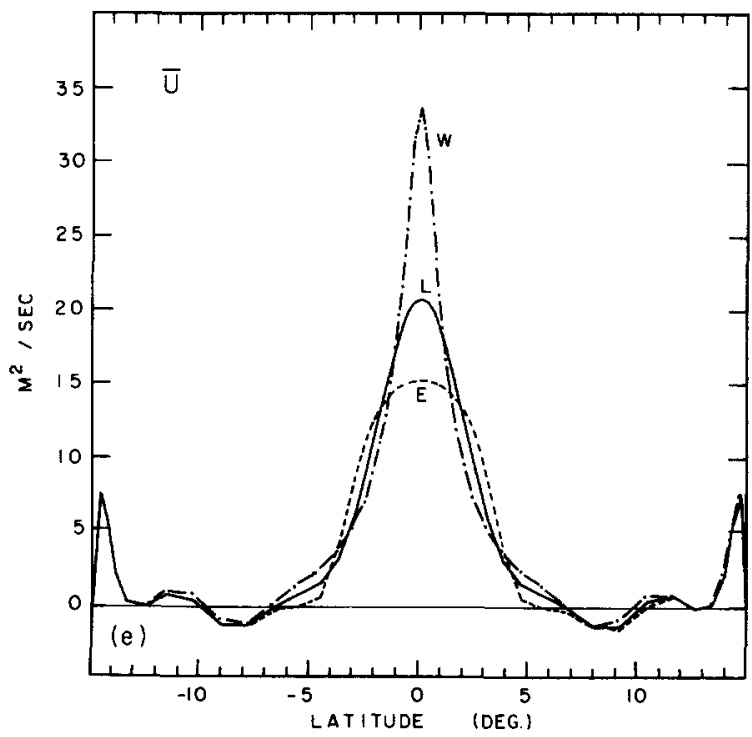

Fig. 3e. Zonal transport, $\bar{u}$. 
As shown in CANE (1979a) the model's vertically integrated transport, $\bar{u}=\eta u^{s}+h u^{1}$, is governed by the shallow water equations. For the $x$-independent case under consideration the solution is the well-known Yoshida (1959) jet (modified only slightly by friction). The linear $\vec{u}$ in Fig. 3e can be compared with the corresponding Yoshida solution shown, for example, in CANE and SARACHIK (1976). (For comparison note that the equatorial length scale is $2.7^{\circ}$, the time scale is 1.78 days, and $\bar{u}$ is scaled by $7 \mathrm{~m}^{2} \mathrm{~s}^{-1}$.) The lower layer velocity $u^{1}$ (Fig. $3 \mathrm{~d}$ ) may be determined from $\bar{u}$ and $u^{\mathrm{s}}$. In a linear inviscid system $u^{1}$ would be zero on the equator; in Fig. 3d the linear $u^{1}(y=0)$ is non-zero only because of the frictional coupling to the upper layer.

Turning now to the nonlinear responses, Figs $3 a$ and $b$ show the kinematic effects of the surface flow. With westerlies (easterlies) the meridional flow in the surface layer is equatorward (poleward) so the equatorial zonal jet is narrowed (broadened) relative to the linear case. At the equator there is a convergence (divergence) of zonal momentum, so the zonal velocity is enhanced (diminished) by nonlinear effects. Figure $3 b$ shows that nonlinear effects have little effect on the magnitude of $v^{s}$, but the inclusion of meridional momentum advection alters the latitude of maximum $v^{s}$. As a result $w_{e}\left(=\eta v_{y}^{s}\right)$ is altered considerably at the equator (Fig. $3 \mathrm{c}$ ); the response to westerlies has a narrow intense downwelling region while with easterlies the upwelling zone is broadened and weakened relative to the linear response.

The effect of downward advection of zonal momentum for the westerly case is apparent in Fig. 3d.* It is clear that $u^{1}$ is large where $w_{e}$ is large. The initial rate of change of $u^{1}$ is proportional to $w_{e} u^{s}$; from the estimates made above this term varies like $t^{3}$ at the equator. Hence $u^{1}$ first grows slowly and then explosively until other effects, such as the zonal pressure gradient, become important. Figure 4 contrasts the evolution of $u^{1}$ in the linear and nonlinear westerly cases. Figure 3 a shows that vertical advection also enhances the vertically integrated transport at the equator [cf. equation (8)]. In summary, the initial effect of nonlinearities is to enhance and narrow both lower layer velocities and total transports for westerlies and to broaden and weaken them for easterlies. Vertical momentum advection is the critical element in the westerly response.

\section{(b) The later stages of evolution}

The long-time evolution of the circulation has been discussed by CANE (1979b); only the points essential to the present discussion will be reviewed here. The principal coastal influence at the equator is to establish a zonal pressure gradient. The gradient travels eastward from the west coast with the Kelvin wave speed and westward from the east coast at the $n=1$ Rossby wave speed (see CANE, 1979b). With westerlies the equatorial zonal jet continues to accelerate until this pressure gradient force arrests its growth. For example, at the central longitude of the model ocean (reached by the Kelvin wave from the west in 10 days) $\bar{u}$ reaches its maximum value at 12 days, after which the zonal pressure gradient causes it to decelerate until day 40 when it approximates its steady state value. The final steady state is characterized by a narrow intense eastward surface flow at the equator and a narrow, less intense eastward subsurface flow. While the westward pressure gradient force

\footnotetext{
* Note that $u^{1}$ for easterlies has become slightly eastward at the equator. This is an artifact of the model's layered structure, which allows $u_{z}$ to be non-zero in the lower layer as soon as $u^{s} \neq 0$, regardless of vertical diffusion. See the discussion of the continuous case given above.
} 

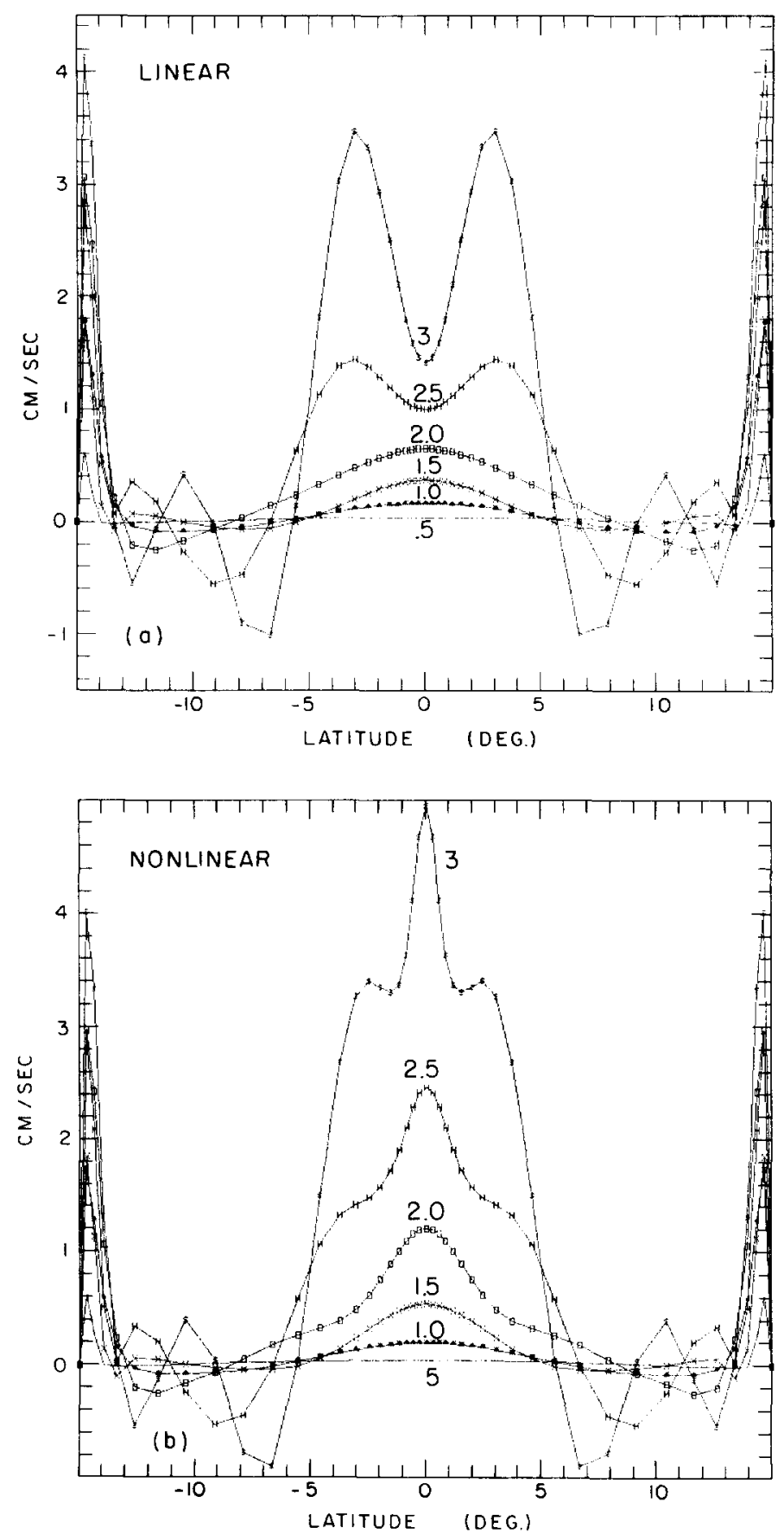

Fig. 4. Meridional sections of $u^{1}$ in response to switched on westerlies. Intervals of 0.5 equatorial time units (0.89 days). (a) Linear. (b) Non-linear. 
set up by the wind slows the subsurface flow, it is insufficient to reverse it in the face of the advection of eastward momentum from the surface layer (see CANE, 1979b, Fig. 14).

With easterlies transport at the equator is ultimately eastward with a strong undercurrent carrying most of the water. The undercurrent is driven by the eastward pressure gradient force and the associated meridional circulation carrying vorticity equatorward, as discussed in Section 2. Initially the transport is westward with the wind and the lower layer flow is weak (Figs 3d, e). It is only after the eastward pressure forces are established that the transport reverses and the undercurrent sets up. Both $u^{1}$ and $\bar{u}$ take about 80 days to reach their maximum values (see CANE, 1979b).

While both cases have eastward subsurface flow the dynamics involved are very different for easterlies and westerlies. For easterlies the zonal pressure gradient is crucial; this involves a basin-wide rearrangement of mass and takes a long time to set up. For westerlies the flow is retarded by the pressure gradient; it is maintained by equatorial downwelling carrying the zonal momentum put in by the wind at the surface.

\section{RESPONSE TO THE RELAXATION OF THE WINDS}

The differences between easterlies and westerlies discussed in the last section have striking dynamical consequences when the winds relax. We again consider two distinct time periods: the initial $x$-independent response to the switch-off of the wind, before coastal influences are felt, and a later stage when coastal effects are present so that the zonal pressure gradient is altered. We will not explicitly consider the linear response; for a linear system the changes in $u$ and $h$ in response to switching off a wind stress $\tau$ are the same as the response of a resting ocean if a wind stress $-\tau$ is turned on. With an initial state that is not at rest the nonlinear changes are generally not well approximated by the resting linear response.

\section{(a) Early stages of relaxation}

Figure 5 a shows the initial response of $u^{1}$ when easterly winds are switched off. Over the first 9 days there is virtually no change in the undercurrent. As the undercurrent is maintained by the zonal pressure gradient and the latter is unaltered, this result could be anticipated. With the shut-off of the winds the surface flow is driven eastward down the pressure gradient so the eastward transport surges.

By contrast, $\boldsymbol{u}^{1}$ decreases rapidly when westerly winds are switched off (Fig. 5b), becoming less than $1 / 3$ of its initial value within 9 days. With the cessation of the wind the surface current rapidly reverses in response to the pressure gradient : $u^{s}$ becomes westward at the equator and $v^{s}$ poleward near the equator. As a result $w_{e}$ at the equator decreases rapidly and then reverses, becoming slightly positive (Fig. 6). This not only cuts off the conduit of eastward momentum to the subsurface layer, but also speeds the change from eastward to westward in the surface layer, as is evident in Fig. 7, which shows the changes in $u^{s}$ from day 0 . Just as in the spin-up of westerlies all processes contributed to eastward acceleration in the lower layer, with relaxation all the processes act to decelerate the subsurface jet. The whole chain of events is frictional, inertial, and independent of changes in the pressure gradient and so can take place locally and rapidly.

While the subsurface current decreased drastically when the winds were turned off it did not reverse as observed at Gan (see Fig. 1; this will be discussed further below). However, 

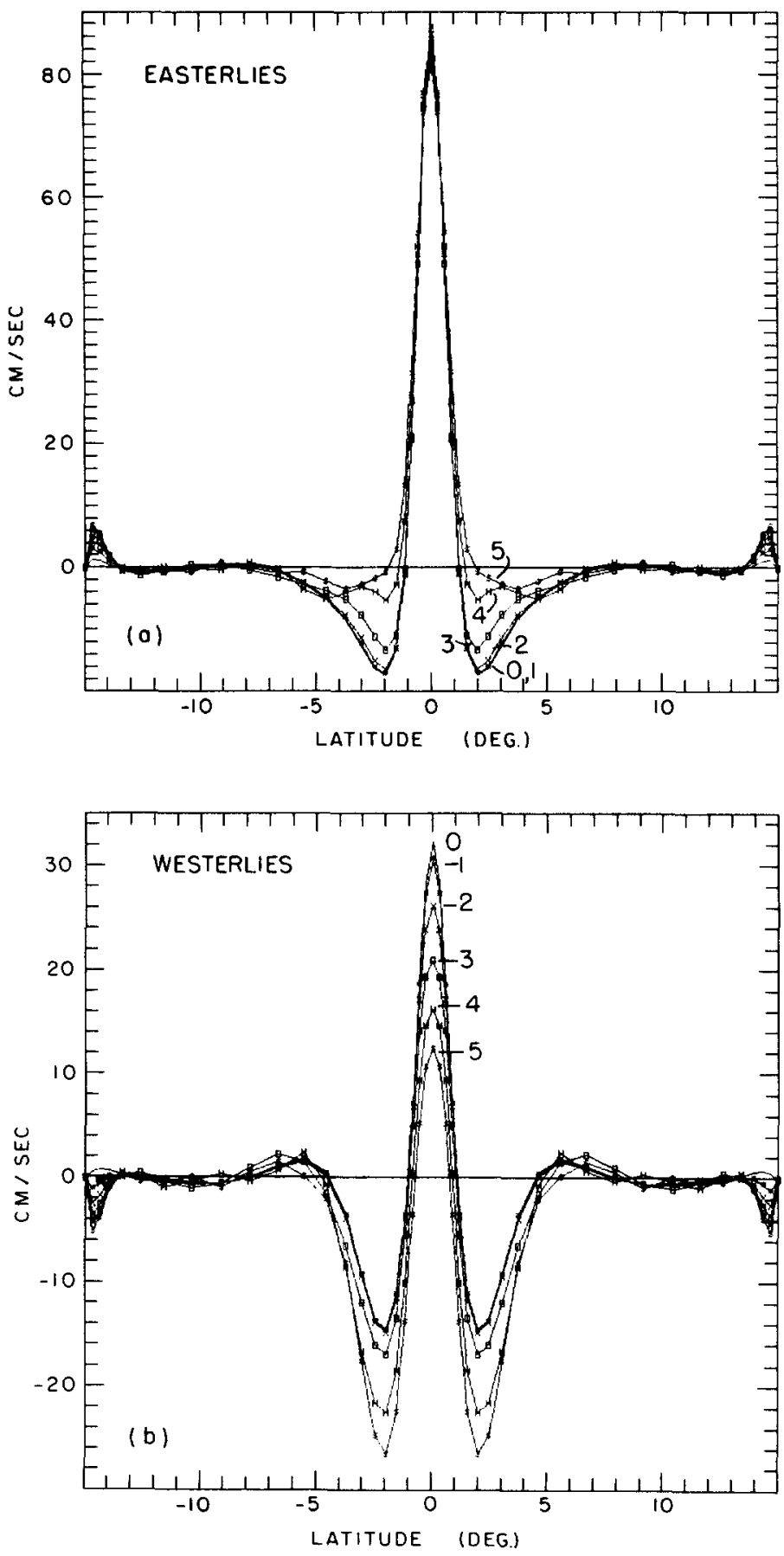

Fig. 5. Meridional sections of $u^{1}$ after the relaxation of the winds. Intervals of one equatorial time unit (1.78 days). (a) Easterlies. (b) Westerlies. 


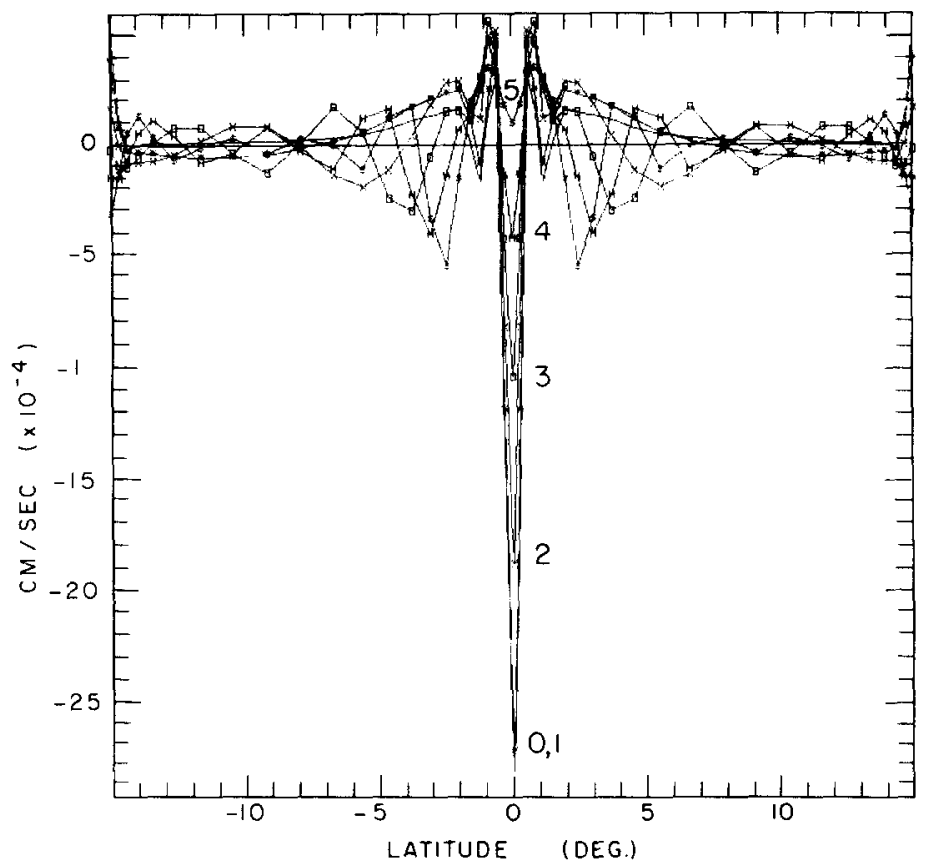

Fig. 6. Meridional sections of $w_{e}$ after the relaxation of westerly winds. Intervals of one equatorial time unit (1.78 days).

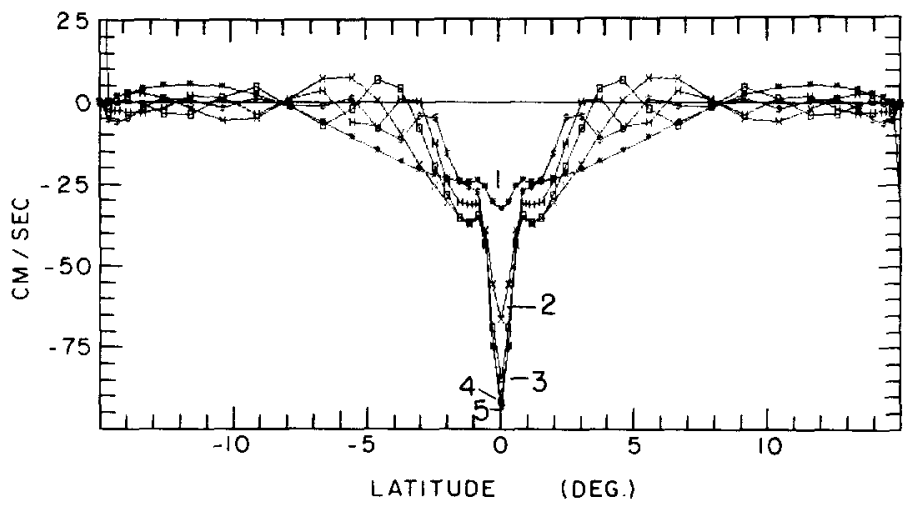

Fig. 7. Meridional sections of the change in $u^{s}$ after the relaxation of westerly winds. Intervals of one equatorial time unit ( 1.78 days).

the winds at Gan did not simply go to zero; rather, they shifted from westerly to northerly. Figure 8 shows what happens in the model if a similar change is made: the current at the equator reverses within 10 days, as in the observations. This comes about because the flow pattern moves upwind (cf. CHARNEY and SPIEGEL, 1971; CANE, 1979b) so that the westward current initially south of the equator becomes the equatorial current. Such an upwind shift would presumably occur in the Indian Ocean in the face of meridional winds. The flow at 


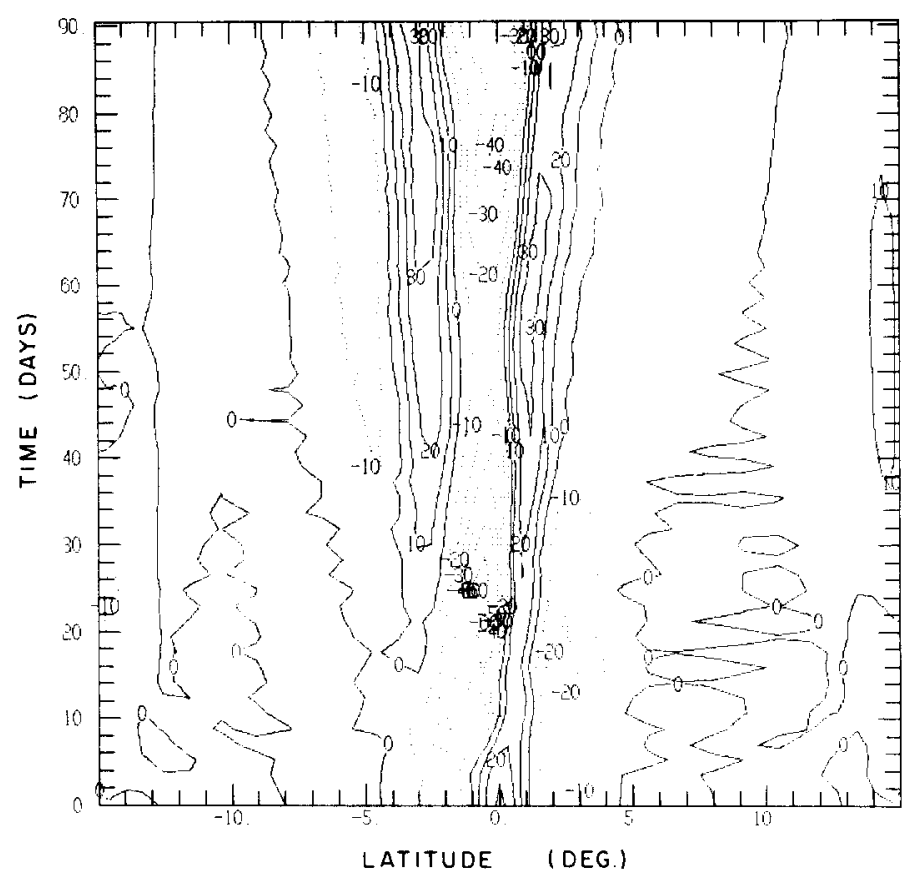

Fig. 8. Time-latitude contours of $u^{1}\left(\mathrm{~cm} \mathrm{~s}^{-1}\right)$ at the central longitude of the basin $(x=14.3)$ after the winds change from westerly to northerly. Upwind displacement reverses the currents at the equator.

the equator would then depend on what currents were initially present south of the equator, but there are no observations of the currents south of Gan.

(b) Later stages of relaxation: coastal effects

While $u^{1}$ at the central longitude of the basin decreased rapidly when the westerlies were turned off (Fig. 5b) it did not reverse. The reason for this is apparent from Fig. 9: the arrival of a Kelvin wave from the western boundary slowed the change in $u^{1}$. Only at the last longitudes in the basin to be affected by the Kelvin or $n=1$ Rossby mode (the region at $x=22$ ) does the velocity become westward. This takes 13 days; these are the only longitudes where the pre-existing westward pressure gradient has enough time to make the currents reverse before being reduced by coastal influences (cf. Fig. 11).

Figure 10 shows the time variation of meridional sections of $u^{1}$ at the center of the basin. After easterlies are switched off (Fig. 10a) the undercurrent is initially unchanged: at day 10 the Kelvin mode from the west reaches this longitude and the strength of the undercurrent begins to drop off. It is the coastal influences that readjust the zonal pressure gradient toward zero (to 'balance' the absence of wind). This reduction of the pressure force driving the undercurrent causes the undercurrent to weaken.

As already noted, the sequence is just the opposite with westerlies; the initial rapid weakening of the subsurface flow is halted by coastal influences, which reduce the westward pressure gradient force towards zero. Interestingly, $u^{1}$ reaches a minimum at about 25 days (at $x=14.3$; Fig. 10b) and then increases again. By day 60 it has nearly reached its initial 


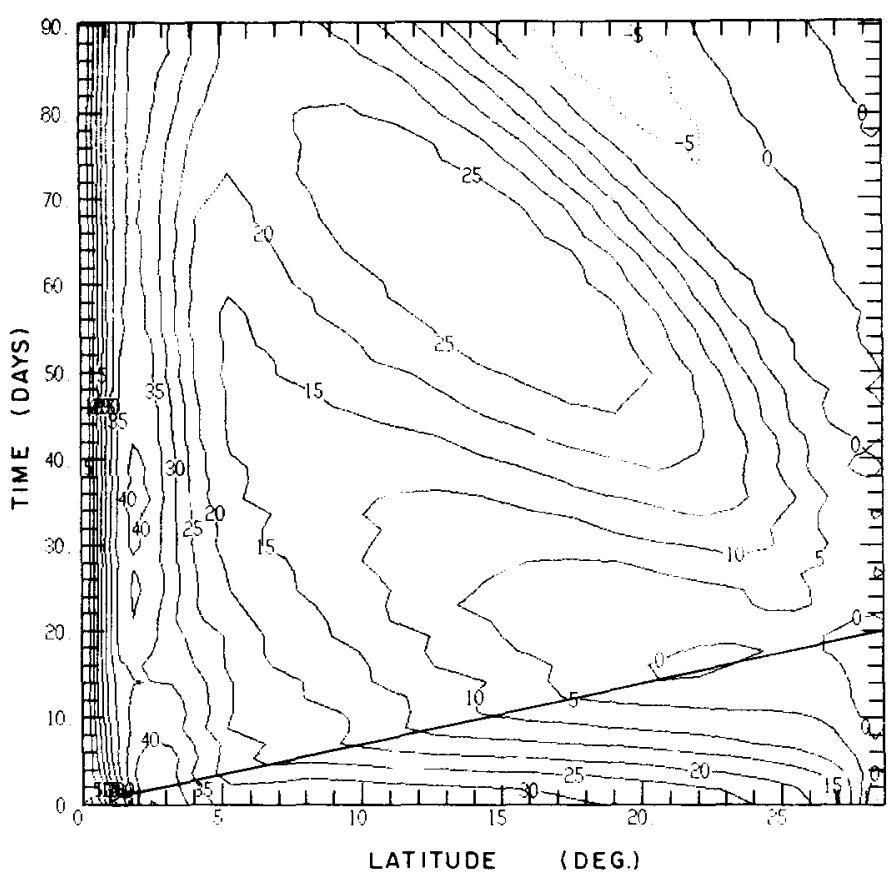

Fig. 9. Contours of $u^{1}$ ( $\mathrm{cm} \mathrm{s}^{-1}$ ) along the equator after the westerly winds relax. The straight line originating at the lower left indicates the Kelvin wave path.

strength. In fact, at this time the region of eastward flow is broader than it was at day 0 so the subsurface transport is greater. The surface flow is weaker than the subsurface flow, in contrast to the initial situation.

The reason for the regeneration of the undercurrent is apparent from Fig. 11, which shows equatorial sections of layer depth deviation at various times. From day 25 onward the pressure gradient force is eastward at $x=14.3$. This gives rise to an equatorial undercurrent dynamically similar to those in the Atlantic and Pacific, as discussed in Section 2. During this period there is weak upwelling at the equator and subsurface meridional convergence (though the flow is not steady as in Section 2). This means that vorticity is transported to the equator, adding to the strength of the undercurrent flowing down the pressure gradient.

The pressure gradient at $x=14.3$ is initially reversed by the Kelvin mode propagating in from the west, but the slope of the dynamic topography begins to steepen more sharply with the arrival of the $n=1$ Rossby wave at day 30 . At day 64 the pressure gradient at $x=14.3$ begins to reverse again and the undercurrent consequently weakens (Fig. 10b).

\section{DISCUSSION AND CONCLUSIONS}

The above results suggest how the relaxation of the winds can generate an undercurrent by causing an adjustment of mass so that the dynamic topography slopes upward to the west. The scant evidence available for the Indian Ocean shows that the dynamic 

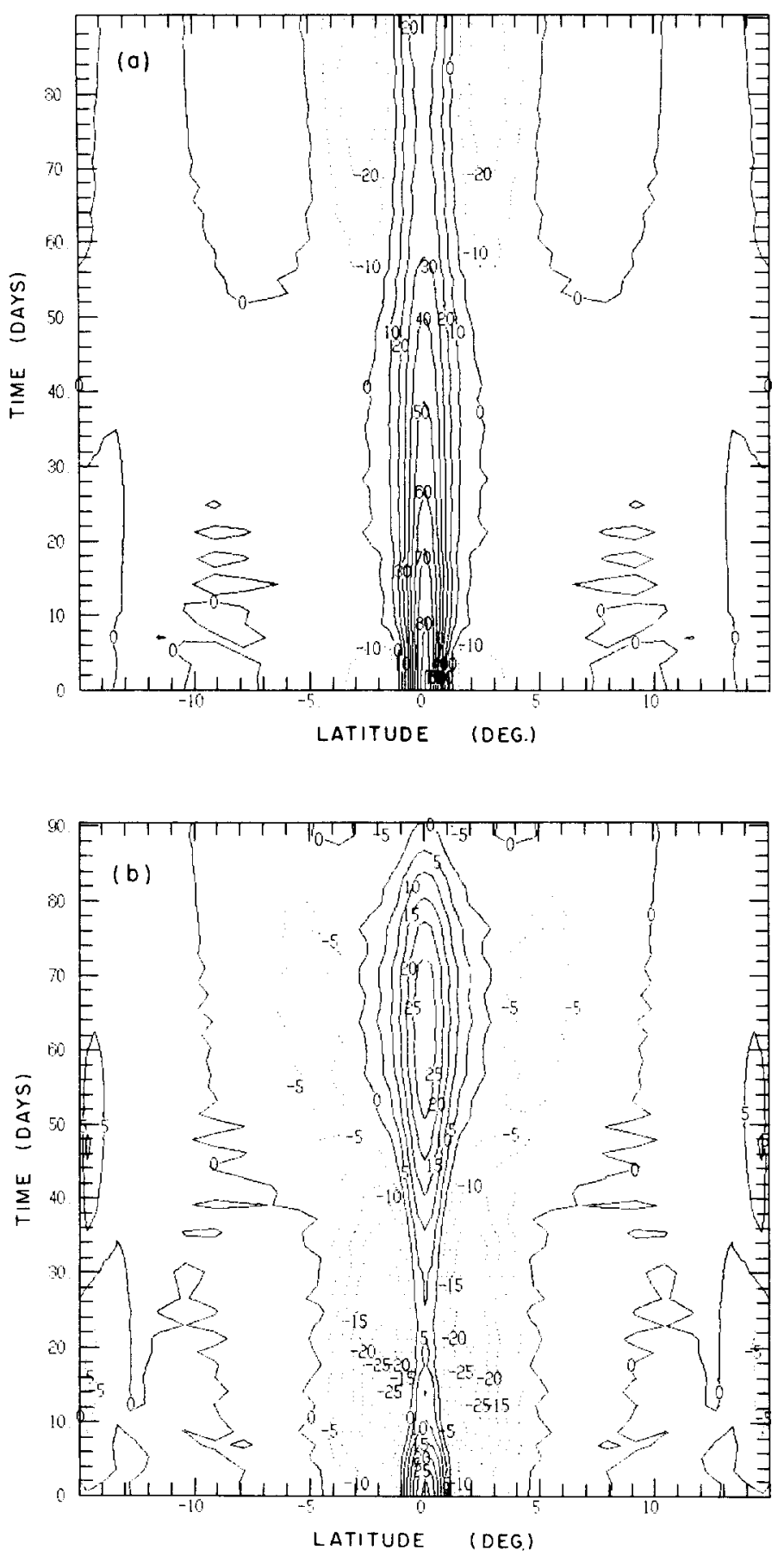

Fig. 10. Time-latitude contours of $u^{1}\left(\mathrm{~cm} \mathrm{~s}^{-1}\right)$ at $x=14.3$ after the relaxation of the winds. (a) Easterlies. (b) Westerlies. 

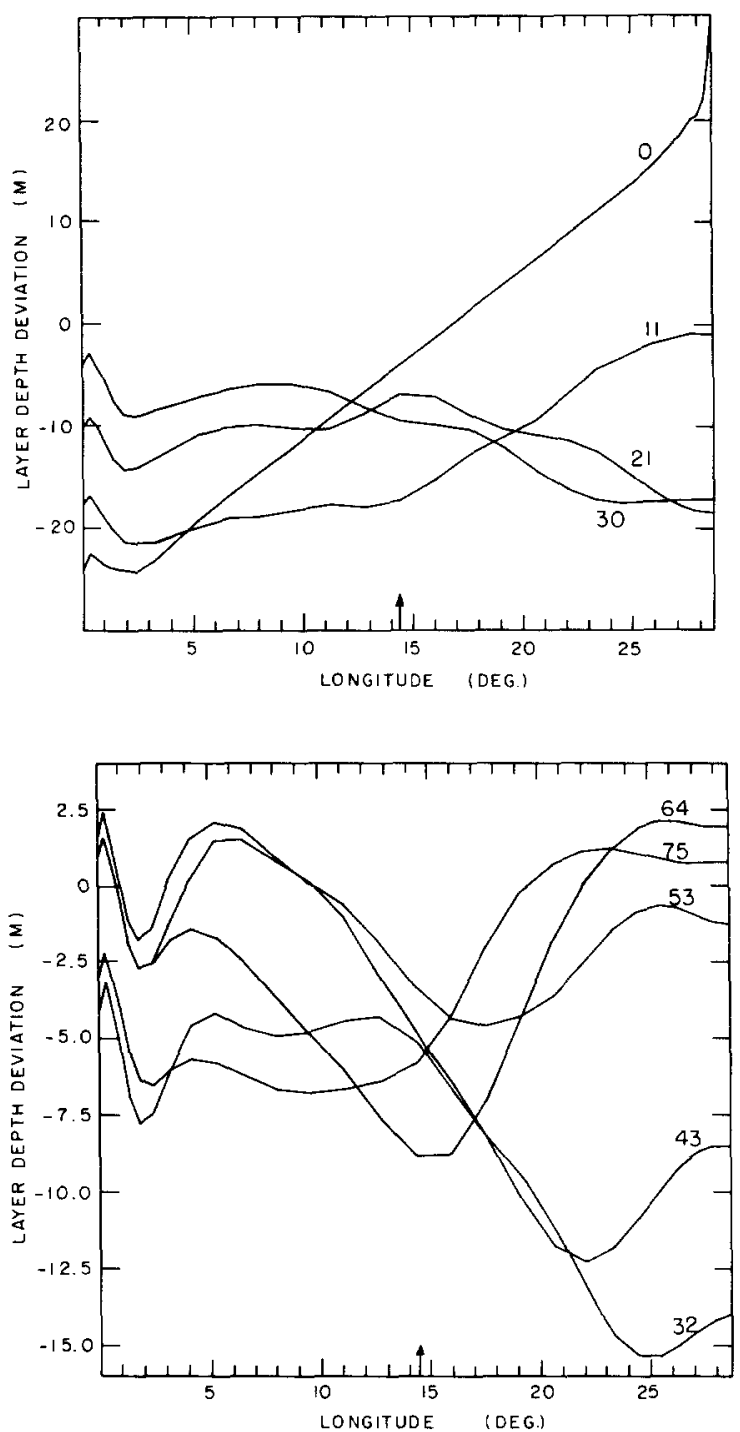

Fig. 11. Sections of layer-depth deviation $h$ along the equator after the relaxation of westerlies. Labelled by time in days. To convert to dynamic topography multiply by $\Delta \rho / \rho=0.002$.

topography at undercurrent depth is always higher to the east except in the early spring (ERIKSEN, 1979, Fig. 5; or see TAFT and Knauss, 1967). As noted in the introduction, this is the only time of year when an undercurrent with the weak surface flow characteristic of the Atlantic and Pacific has been observed in the Indian Ocean.

The presence of the undercurrent in March 1973 and its absence in March 1974 merit comment. The striking difference between the two in the wind record at Gan (Fig. 1) is in the last quarter of the preceding year; in 1972 the winds remained weak from October through December, while in 1973 there were strong westerlies throughout this period, postponing the relaxation until January 1974. Ten years of wind data at Gan (R. A. KNOx, 
private communication) show that neither one pattern nor the other occurs with sufficient frequency to be considered typical. It is perhaps worth noting that 1963, the year preceding Swallow's (1967) undercurrent observations, was similar to 1972. [Wind data are not available for 1962, preceding the Lusiad expedition (TAFT and KNAUSS, 1967).]

We may translate adjustment times for the model to the Indian Ocean as follows: First, note that although the currents on the equator are highly nonlinear the wave speeds observed in the model are the same as those given by linear theory (at least for the most important modes for the adjustment process, the Kelvin and $n=1$ Rossby waves). Also, while the zonal currents do not resemble their linear counterparts the deviations of the layer depth do. Hence, we may adjust the time scales linearly. The zonal extent of the Indian Ocean is twice that of the model basin with Gan roughly at the midpoint. The Kelvin wave speeds for the first and second baroclinic modes are 2.8 and $1.8 \mathrm{~m} \mathrm{~s}^{-1}$, respectively [based on ERIKSEN's (1979) density profiles]; for the model it is $1.9 \mathrm{~m} \mathrm{~s}^{-1}$. Hence if the model undercurrent at the center of the basin reaches a maximum two months after the wind is relaxed, one would expect the corresponding time for Gan to be either three or four months depending on which baroclinic mode governed. Both the structure of the undercurrent and the fact that in the spring the slope of the dynamic topography at 0 dbar is opposite to that at 75 to 100 dbar (TAFT and KNAUSS, 1967) imply that the second baroclinic mode is dominant. This is confirmed by PHILANDER and PaCanowski's (1980) recent numerical study. The implication of all of this is that for an undercurrent to be present at Gan in late February to early March the winds in the equatorial Indian Ocean would have to relax in the preceding October - at about the time of the monsoon transition.

It must be remembered that the data in Fig. 1 give the winds only at a single point and that, unlike the local dynamics discussed earlier, changes in the zonal pressure gradient involve the winds over the entire length of the ocean within (at least) a few degrees of the equator. The suggested explanation for the differences between 1973 and 1974 is offered only as a hypothesis. It would gain support if evidence existed to show that in those years the winds over some significant fraction of the Indian Ocean behaved like the winds measured at Gan.

The data collected by KNox (1976) are extremely valuable because they are the only long time series of simultaneous wind and current measurements in the equatorial Indian Ocean. They are, however, data from only a single point and it is quite possible that unknown features of the winds or currents might affect the applicability of our results to the Indian Ocean. We can only conclude that the following features are dynamically reasonable and are consistent with available observations:

(1) The equatorial response to westerlies is predominantly local and rapid, occurring on an inertial-frictional timescale.

(2) The dominant response to easterlies is non-local and not rapid. The relevant timescale is the time needed to set up a zonal pressure gradient, a wave travel time.

(3) The differences in the ocean's response to easterlies and westerlies mean that nonlinearity is a significant influence.

(4) Westerlies give subsurface eastward flow via vertical advection of momentum. This dominates over the influence of the westward pressure force that such winds create.

(5) Easterlies give an eastward pressure gradient force that, both directly and by meridional advection of vorticity, drives an undercurrent.

(6) The relaxation of westerlies gives rise to an eastward pressure gradient force. This can drive an undercurrent. 
The last point is consistent with the differences between March 1973 and 1974, but better observations of winds, dynamic topography, and currents are needed to test the idea properly. While the localness of some of the dynamical ideas given above mean they can be tested if only the local winds are known, any detailed comparisons require some knowledge of oceanographic conditions in the vicinity, including information about off-equatorial currents.

Acknowledgements-My thanks to R. A. KNox for generously making available his unpublished data. Thanks to CHARLES ERIKSEN for supplying equivalent depths appropriate to the Indian Ocean. Valuable discussions with George Philander, E. S. Sarachik, and Laura CANe are gratefully acknowledged. Special thanks to Manina ALMEIDA for assistance with the computer runs and to VIRGINIA MILLS for careful preparation of the manuscript. The final stages of manuscript preparation were supported by NASA Grant NGR 22-009-727.

\section{REFERENCES}

CANE M. A. (1979a) The response of an equatorial ocean to simple wind stress patterns: I. Model formulation and analytic results. Journal of Marine Research, 37, 233-252.

CANE M. A. (1979b) The response of an equatorial ocean to simple wind stress patterns: II. Numerical results. Journal of Marine Research, 37, 253-299.

CANE M. A. and E. S. SARACHIK (1976) Forced baroclinic ocean motions: I. The linear equatorial unbounded case. Journal of Marine Research, 34, 629-665.

Charney J. G. (1960) Non-linear theory of a wind-driven homogeneous layer near the equator. Deep-Sea Research, 6, 303-310.

Charney J. G. and S. SPIEGEl (1971) Structure of wind driven equatorial currents in homogeneous oceans. Journal of Physical Oceanography, 1, 149-160.

ERICKSEN C. C. (1979) An equatorial transect of the Indian Ocean. Journal of Marine Research, 37, 215 --232.

FofonofF N. P. and R. B. MONTGOMERY (1955) The equatorial undercurrent in the light of the vorticity equation. Tellus, 7, 518-521.

Gill A. E. (1975) Models of equatorial currents. In Proceedings of the Symposium on Numerical Models of Ocean Circulation, Washington, National Academy of Science, $364 \mathrm{pp}$.

HIDE R. (1969) Dynamics of the atmospheres of the major planets with an appendix on the viscous boundary layer at the rigid bounding surface of an electrically-conducting rotating fluid in the presence of a magnetic field. Journal of the Atmospheric Sciences, 26, 841-853.

KNox R. A. (1976) On a long series of measurement of Indian Ocean equatorial currents near Addu Atoll. DeepSea Research, 23, 211-221.

O'Brien J. J. and H. E. Hurlburt 1974 An equatorial jet in the Indian Ocean, Theory. Science, 194, $1075-1077$.

Philander S. G. H. (1973) Equatorial undercurrent: measurements and theories. Reviews of Geophysics, 11, $513-570$

Philander S. G. H. (1979) Non-linear coastal and equatorial jets. Journal of Physical Oceanography, 9, $739-747$.

Philander S. G. H. and R. PAcinowski (1980) The generation of equatorial currents. Journal of Geophysical Research, 85, 1123-1136.

Robinson A. R. (1966) An investigation into the wind as the cause of the Equatorial Undercurrent. Journal of Marine Research, 24, 179-204.

Swallow J. C. (1967) The equatorial undercurrent in the western Indian Ocean in 1964. Studies in Tropical Oceanography, 5, 15-36, Univ. of Miami Press, Florida.

TAFT B. A. and J. A. KNAuss (1967) The equatorial undercurrent of the Indian Ocean as observed by the Lusiad expedition. Bulletin of the Scripps Institution of Oceanography, 9, $163 \mathrm{pp}$.

Yoshida K. (1959) A theory of the Cromwell current and of the equatorial upwelling. Journal of the Oceanographical Society of Japan, 15, 154-170. 